\title{
Power Communication Optical Network Traffic Grooming Algorithm Based on Differentiated Services
}

\author{
Zhong-miao KANG ${ }^{1}, \mathrm{Xi-jie} \mathrm{DONG}{ }^{2}$, Hui-tong LIU $^{3}$ and Liang-rui \\ ${ }^{1}$ Power Dispatch and Control Center of Guangdong Power Grid \\ Guangzhou, China \\ ${ }^{2}$ State Grid Information \& Telecommunication BranchBeijing, China \\ ${ }^{3,4}$ North China Electric Power UniversityBeijing, China \\ *Hui-tong LIU
}

\begin{abstract}
Keywords: Power Communication Optical Network, Differentiated Services, Traffic Grooming.
\end{abstract}

\begin{abstract}
With the development of smart grid, the data service of large particle and IP in power communication network has been increasing, which has put forward a great challenge to the existing power communication optical network based on MSTP (Multi-Service Transfer Platform) technology and WDM (Wavelength Division Multiplexing) technology. Considering the difference of services in the power communication optical network, this paper proposed a traffic grooming algorithm based on differentiated services. The algorithm is based on the layered exchange virtual topology model, and dynamically updates the link cost according to the current available resources of the network. According to the level of groomed service, we use the $\mathrm{K}$ shortest path algorithm based on the O/E/O (Optical/Electrical/Optical) conversion limit to determine the alternative path set and select optimization factors through the traffic grooming path. Under the condition of satisfying the $\mathrm{O} / \mathrm{E} / \mathrm{O}$ conversion restrictions, guide the service to the path which meets its transmission delay requirements and has smaller number of $\mathrm{O} / \mathrm{E} / \mathrm{O}$ transitions, coordinate the distribution of network resources among different levels of service.
\end{abstract}

\section{Introduction}

With the fast and efficient construction of smart grid, the communication network of electric power system has been proposed higher expectations. According to the characteristics of large granularity and IP in the power communication service, optical network traffic grooming technology get into people's horizons when use the advanced optical network technology to carry out the power communications service. The traffic grooming technology converges multiple low-speed traffic streams to one wavelength under the background of a sharp increase in the single wavelength capacity, to realize the high speed data transmission technology, we can achieve the goal of minimizing the use of terminal apparatus and optical/electrical switching equipment through this technical means, therefore it is of great significance to do the research in the power communication optical network traffic grooming field. 
A grooming algorithm based on the QoS (Quality of Service) is proposed in the paper [1], which is to minimize the number of hops and balancing the network load. The end-to-end delay is an important parameter to measure the quality of network service. The main reason for the delay in optical network is the $\mathrm{O} / \mathrm{E} / \mathrm{O}$ conversion times in the path, therefore the time delay constraints can be translated to the $\mathrm{O} / \mathrm{E} / \mathrm{O}$ conversion time constraints of the grooming path when the differentiated service policy is implemented. In the paper [2], the problem of the constrained end-to-end delay is studied, and the corresponding ILP (Integer Linear Programming) conditions are described. The proposed relaxation algorithm based on ILP can achieve better results when it is used in a large scale network. In the paper [3], the problem of multicast traffic grooming is studied. In order to improve the success rate of the high priority traffic grooming, the traffic grooming algorithm based on preemption is proposed in the paper [4]. However, the problem of this algorithm is that when the network is in a high load condition, if the bandwidth requirements of high priority traffic demand is higher, it cannot be groomed on a single link even if use the preemption mechanism. In order to solve the problem of high blocking rate of large granularity traffic, the traffic grooming algorithm based on traffic splitting mechanism is proposed in the paper [5]. The traffic splitting mechanism can be divided into many small particles service, each service can reach the destination node through different channel. But the problem of this algorithm is that it has a high requirement for the exchange node's cache, and the existing cache technology cannot meet the requirements of the service.

Considering that the results in the past just focused on reducing the traffic delay, this paper proposed a dynamic traffic grooming algorithm which can distinguish the traffic delay. Through coordinating the resources among the service with different delay requirements, and making full use of the protected resources, it can not only guarantee the blocking rate and delay performance, but also improve the resource utilization.

\section{Hierarchical Interconnection Virtual Topology Model Based on Differentiated Services}

According to the service bandwidth requirements, the service of power communication optical network can be roughly divided into three categories: the service which the bandwidth requirement is $64 \mathrm{~kb} / \mathrm{s}$ and below, the service which the bandwidth requirement is between $64 \mathrm{~kb} / \mathrm{s}$ to $2 \mathrm{Mb} / \mathrm{s}$ and the service which the bandwidth requirement is $2 \mathrm{Mb} / \mathrm{s}$ or more. In the research of the traffic grooming algorithm, the bandwidth requirement index can be used as the limiting condition for constructing the dynamic traffic grooming model. According to the transmission delay index, the service of power communication optical network can be divided into three categories: time delay sensitive services $G_{0}$, second delay sensitive services $G_{1}$ and non real-time services $G_{2}$, the service priority decreases in turn. The transmission delay index can evaluate the transmission performance of the service well, so it can be used as the differentiation index of the service in power communication optical network. 
Given the physical topology of optical transport network $\mathrm{G}(V, E, T, W, N)$, where $V$ expresses the nodes set in the topology, $E$ expresses the connecting edge set in the topology, $T$ expresses the tunable optical transceiver set for each node, $W$ is expressed in available wavelength set for each fiber. When the service request $R\left(V_{s}, V_{d}, b w\right.$, grade $)$ arrives, determine the request level grade of the access service, source nodes $V_{s}$ and destination nodes $V_{d}$, and the service request bandwidth $b w$. Nodes in the physical topology $G$ include the source node $V_{s}$, the destination node $V_{d}$ and other intermediate nodes, divide the corresponding layered exchange edge into the source \& destination node layered exchange edge and middle node layered exchange edge in the layered exchange virtual topology; when the optical path consists of the wavelength link in the same plane exists in the topology, the direct light path can be directly established, and delete the wavelength links of this direct path in the virtual topology at the same time, complete the process of network resources occupation. The number of tunable optical transceivers for each node is $|T|$, update the number of optical transceivers in real time according to the network traffic. When the number of tunable optical transceivers is exhausted, the service through the node can no longer use the wavelength link of the node, traffic grooming can be carried out only through the existing straight optical path of the node, therefore, it is required to remove the wavelength link between the node and the other nodes. Finally, form a service differentiating layered exchange virtual topology through wavelength link, straight-through link, layered exchange edge, and each virtual node.

Hypothesis the edge $e$ in the layered exchange virtual topology based on differentiated services can be expressed as $e_{m m}^{\lambda_{i}}\left(t y p e, \lambda_{i}, w_{e}, b w_{\text {free }}\right)$, where type expresses the type of the edge $e$, namely the corresponding type of wavelength links, straight-through links and layered exchange edges are $1,2,3, \lambda_{i}$ expresses the wavelength plane at which the edge is located, $w_{e}$ expresses the corresponding link costs, $b w_{\text {free }}$ expresses the remaining bandwidth of the edge. Link costs of edge $e$ is,

$$
w_{e}=\left\{\begin{array}{cl}
|W| / c, & \text { type }=1 \\
1, & \text { type }=2 \\
0, & \text { type }=3
\end{array}\right.
$$

where for wavelength link $e(t y p e=1), c$ expresses the number of available wavelengths in the physical link corresponding to the wavelength link $e$ in the physical topology, the initial value of $w_{e}$ is 1 , namely the number of available wavelengths $c=|W|$ when there is no connection request. The corresponding $w_{e}$ value of straight-through link $e($ type $=2)$ is 1 . For the node layered exchange edge $e($ type $=3)$, the link cost is 0 . The cost of the path from the source node $V_{s}$ to the sink node $V_{d}$ is,

$$
f_{\left(V_{s}, V_{d}\right)}(e)=\sum_{e \in p a t h} w_{e}
$$


where path express a route from the source node $V_{s}$ to the sink node $V_{d}$.

\section{Description of DSTGA Traffic Grooming Algorithm}

Before implementing the service grooming strategies, compute the service counseling alternative path set of different service in power communication optical network using the classic $\mathrm{K}$ shortest path algorithm yen's K-shortest paths ${ }^{[8]}$ based on the service differentiating layered exchange virtual topology, which can be the greatest degree of satisfaction with the selection requirements of service to different paths and improve the traffic carrying capacity of the overall network.

1) When the grooming service is the delay sensitive service $G_{0}$, the path selection optimization factor $\alpha_{0}$ is defined as,

$$
\alpha_{0}=\left\{\begin{array}{lc}
\frac{b w_{\text {free }}}{C_{0}}, & b \leq b w_{\text {free }}<C_{0} \\
\frac{b}{C_{0}}-\varepsilon, & b w_{\text {free }}=C_{0}
\end{array}\right.
$$

where $b$ expresses the traffic grooming bandwidth request, $b w_{\text {free }}$ expresses the remaining bottlenecks bandwidth of alternative paths, $C_{0}$ expresses the maximum transmission bandwidth of wavelength links, $b \leq b w_{\text {free }}<C_{0}$ expresses the path constituted for direct links by alternative paths, $b w_{\text {free }}=C_{0}$ expresses the path constituted for wavelength links by alternative paths. $\varepsilon$ expresses an infinitely small parameter.

Give priority to the establishment of service connection path through the existing straight-through link of the virtual topology to meet the needs of service bandwidth. If it cannot be achieved, the optical path will be established by using the idle wavelength link in the wavelength plane of the virtual topology graph.

2) When the grooming service is the second delay sensitive services $G_{1}$, the path selection optimization factor $\alpha_{1}$ is defined as,

$$
\alpha_{1}=\left\{\begin{array}{cc}
\frac{b w_{\text {free }}}{C_{0}}, & b \leq b w_{\text {free }}<C_{0} \\
\frac{b}{C_{0}}-\varepsilon, & b w_{\text {free }}=C_{0} \\
\left(\frac{b}{C_{0}}-2 \varepsilon\right) * \frac{b w_{\text {free }}}{C_{0}}, & b \leq b w_{\text {free }}<C_{0} \text { and there is at least one link } b w_{\text {free }}=C_{0}
\end{array}\right.
$$

where $b \leq b w_{\text {free }}<C_{0}$ and there is at least one link $b w_{\text {free }}=C_{0}$ express the path mixed constituted for direct links and wavelength links by alternative paths.

Give priority to the establishment of service connection path through the existing direct link of the virtual topology to meet the needs of service bandwidth. If unable to successfully establish a path, the optical path will be established by using the idle 
wavelength link in the wavelength plane of the virtual topology graph. When the service is unable to complete the grooming, use the idle wavelength link, the straight path and the layered exchange edge to construct the multi-hop traffic grooming path.

3) When the grooming service is the non real-time service $G_{2}$, the path selection optimization factor $\alpha_{2}$ is defined as,

$$
\alpha_{1}=\left\{\begin{array}{lc}
\frac{b w_{\text {free }}}{C_{0}}, & b \leq b w_{\text {free }}<C_{0} \\
\frac{b}{C_{0}}-2 \varepsilon, & b w_{\text {free }}=C_{0} \\
\frac{b}{C_{0}}-\varepsilon, & b \leq b w_{\text {free }}<C_{0} \text { and there is at least one link } b w_{\text {free }}=C_{0}
\end{array}\right.
$$

Give priority to the establishment of service connection path through the existing straight-through link and layered exchange edge of the virtual topology to meet the needs of service bandwidth. If unable to complete the service counseling, use the idle wavelength link, the straight-through light path and the layered exchange edge to construct the multi-hop traffic grooming path. When the path is not successfully established, the optical path will be established by using the idle wavelength link in the wavelength plane of the virtual topology graph.

When the service access request is $R\left(V_{s}, V_{d}, b w, G_{i}\right)$, the path selection optimization factor is used as the optimization goal of the alternative path set, the specific optimization function is,

$$
F_{\text {opt }}=\max \left(\alpha_{i}\right)
$$

where $\alpha_{i}$ is the path selection optimization factor for all paths in an alternative path set for service $G_{i}$.

The value of $F_{o p t}$ is obtained by using the formula (6), the service connection request is optimized on the corresponding optimal path, and complete the allocation of wavelength, bandwidth, and optical transceiver resources at the same time.

\section{Simulation Results and Analysis}

This paper used the network topology of Natural Science Foundation (NSFNET) in the United States as shown in Figure 1 to simulate the network topology, a total of 14 nodes and 21 two-way physical links. The simulation process used the simulation environment based on events occurrence, the service connection requests $R\left(V_{s}, V_{d}, b w\right.$, grade $)$ are two-way service, source and destination nodes of service request are randomly selected in the node set, the maximum number $|w|$ of wavelengths in each fiber is set to 14 . Single wave length is set to 10 units, the number of single node optical transceivers is $|T|$. The bandwidth of the service demand is in the uniform distribution between 1 and 6 units. According to the actual service volume of each level of power communication service, the optical path connection request arrival rate for three level 
services is set as $\beta_{0}: \beta_{1}: \beta_{2}=1: 2: 7$. In order to facilitate the simulation comparison, the duration of each level service is set to the same, as $\mu_{i}=1(\mathrm{i}=0,1,2)$. The total service load is defined as $\rho=\beta / \mu$ (Erl), where $\beta=\beta_{0}+\beta_{2}+\beta_{2}, \mu=\mu_{i}$.

In this paper, we select the TDGS algorithm proposed in the paper [9], and the MTGA algorithm proposed by paper [10] to compare with the traffic grooming algorithm based on differentiated services (DSTGA) proposed in this paper.

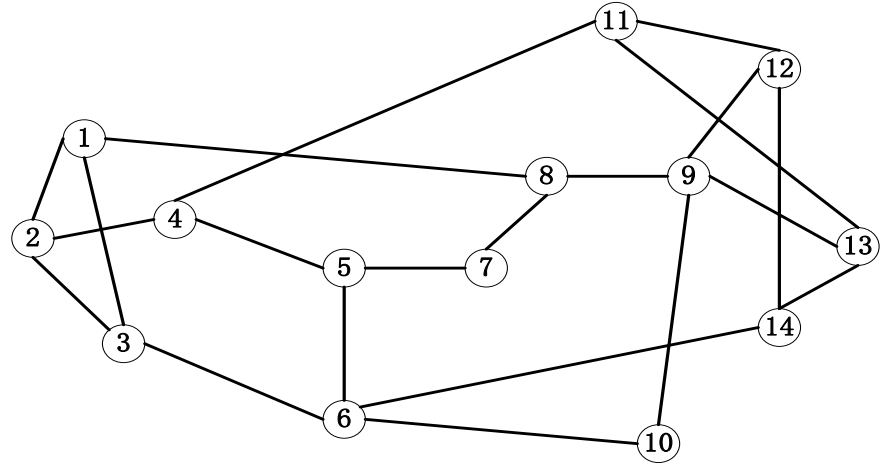

Fig. 1 the United States Natural Science Foundation of China (NSFNET) network topology

\section{Analysis of The Rate of Average Blocking Network}

As shown in Figure 2, the average blocking rate of the three algorithms gradually increases with the increase of the traffic load, but the average blocking rate of the DSTGA algorithm proposed in this paper is significantly lower than that of the MTGA algorithm and the TDGS algorithm. This shows that the average blocking performance of the DSTGA algorithm has a great advantage compared with the other two algorithms.

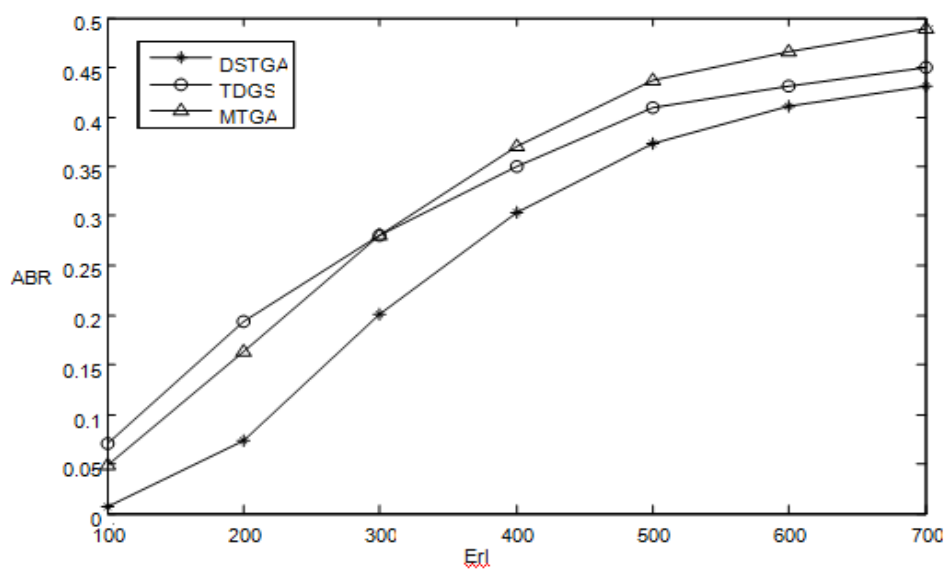

Fig. 2 average blocking rate(ABR) of network

\section{Analysis of Traffic Congestion in Different Grades}

As shown in Figure 3, for the blocking rate of the $G_{0}$ class service, the blocking rate of DSTGA algorithm is significantly lower than MTGA, but it is higher compared with the TDGS algorithm. Considering Figure 2, Figure 3, Figure 4 and Figure 5, it can be found that the TDGS algorithm reduces the blocking rate of the high priority traffic of $G_{0}$ on the basis of sacrificing the blocking performance of $G_{2}$ and $G_{1}$ level traffic as 
well as the average blocking performance of the network, clearly, the algorithm does not have the practical value in the actual operation of the network. As shown in Figure 4, for the blocking rate of $G_{1}$ class, the blocking rate of DSTGA algorithm is better than that of MTGA algorithm and TDGS algorithm under different traffic load intensity. The improvement of the blocking rate of $G_{1}$ grade service by DSTGA algorithm was the most obvious, especially in the medium intensity service load, which is due to the DSTGA algorithm reasonably coordinating the use of resources between the three priorities of the service. But when the traffic load is high, the improvement effect of blocking rate is not significant. This is because in high load conditions, the main reason for the traffic congestion is the lack of wavelength resources and the limitation of $\mathrm{O} / \mathrm{E} / \mathrm{O}$ conversion, at this time, the algorithm is limited to improve the blocking performance. For the blocking rate of $G_{2}$ level traffic, it can be seen from Figure 5 that the blocking performance of $G_{2}$ class of DSTGA algorithm has been improved obviously, especially compared with TDGS algorithm, the blocking rate is obviously reduced. Under different traffic load, the higher the priority of service, the lower the blocking rate, this meets the requirement of different levels of service to the blocking performance.

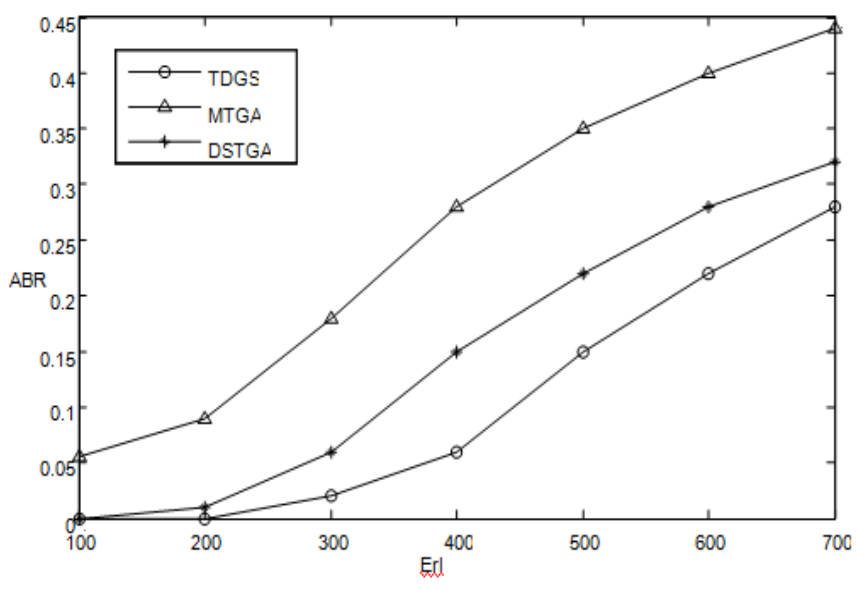

Fig. 3 Comparison of $\mathrm{G}_{0}$ rating traffic blocking rate

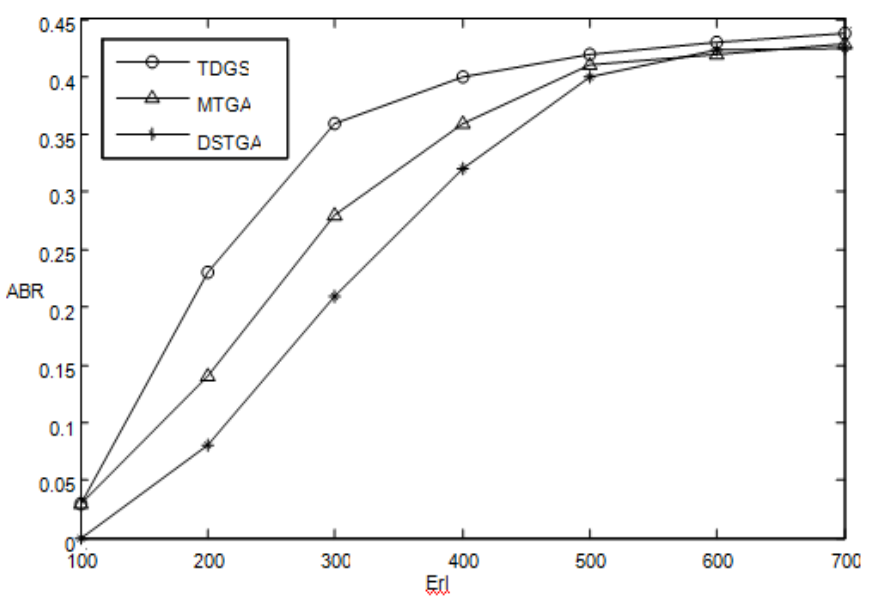

Fig. 4 Comparison of $\mathrm{G}_{1}$ rating traffic blocking rate 


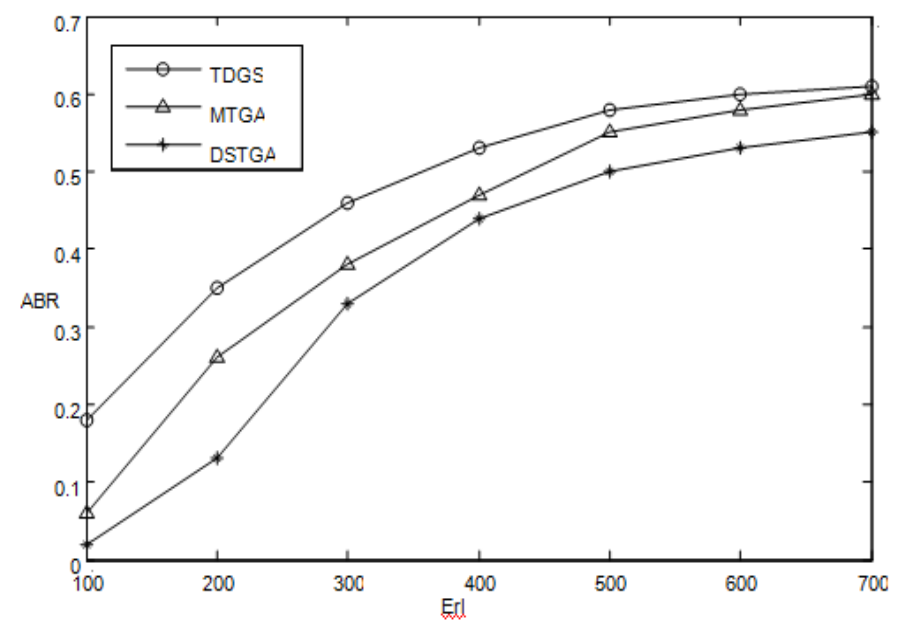

Fig. 5 Comparison of $\mathrm{G}_{2}$ rating traffic blocking rate

From the above analysis, it can be concluded that in the power communication optical network, DSTGA algorithm is better than MTGA algorithm and TDGS algorithm in terms of blocking performance and network resource allocation. The simulation results show that in electric power optical communication network, the DSTGA algorithm proposed in this paper optimized the blocking performance of the whole network and the usage of network resource under the condition of satisfying the difference requirement of the blocking performance of the power communication service.

\section{Conclusion}

This paper considered the dynamic change information of optical network source, proposed an optical network traffic grooming algorithm that is based on differentiated services and established service differentiating model in the power communication optical network. According to the level of groomed service, calculate the alternative path set using the $\mathrm{K}$ shortest path algorithm based on the O/E/O conversion limit, to divert service to the path that meets the requirements of its transmission delay and has smaller number of $\mathrm{O} / \mathrm{E} / \mathrm{O}$ transitions through the service grooming path selection optimization factor, coordinate the distribution of network resources among different levels of service. Simulation results show that the proposed algorithm can effectively reduce the network blocking rate and make full use of the network resources compared with other traffic grooming algorithms.

\section{Acknowledgment}

The work is supported by "The National High Technology Research and Development of China 863 Program (2014AA01A701), the Beijing Natural Science Foundation (4142049) and the National Natural Science Foundation of China (Grant No.51507063)" 


\section{References}

[1] Yen H H, Lee S S W, Mukherjee B. Traffic grooming and delay constrained multicast routing in IP over WDM networks. International Conference on Communications. IEEE, 2008: 5246-5251

[2] Sahasrabuddhe L H, Mukherjee B. Light trees:optical multicasting for improved performance in wavelength routed networks. Communications Magazine, IEEE, 1999, 37(2): $67-73$

[3] Salvadori E, Battiti R. Quality of service in IP over WDM: considering both service differentiation and transmission quality. International Conference on Communications, IEEE, 2004, 3: 1836-1840.

[4] Cheng B, Wang H. A new dynamic traffic grooming algorithm. Optical communication technology, 2009, 33(1): 22-25

[5] Wang J, Yang X, Huang S, et al. An enhanced multi-priority traffic-grooming scheme based on traffic-partition for IP-over-WDM networks. International Society for Optics and Photonics Asia-Pacific Optical Communications, 2007: 678438

[6] Fawaz W, Daheb B, Audouin O. Service Level Agreement and Provisioning in Optical Networks. Communications Magazine, IEEE, 2004, 42(1): 36-43

[7] Xu T, Ding X L, Li J F. KA summary of the shortest path algorithm.Computer engineering and design,2013,34(11):3900-3906

[8] Yen J Y. Finding the k shortest loopless paths in a network. management Science, 1971, 17(11): 712-716

[9] Li J, Xiao H, Wang J. A new multi-priority grooming scheme of WDM optical networks. International Conference on Computer Science and Network Technology (ICCSNT), IEEE, 2012: 1418-1421

[10] Hui Y J, Jin H, Fan G. Traffic grooming algorithm for multi priority QoS . Optical communication research, 2010(2): 13-16 